\title{
FIXED POINT THEOREMS VIA FAMILY OF MAPS IN WEAK NON-ARCHIMEDEAN MENGER PM-SPACES
}

\author{
DeEpaK Singh $^{\mathrm{a}}$ And Amin Ahmed ${ }^{\mathrm{b}, *}$
}

\begin{abstract}
C. Vetro [4] gave the concept of weak non-Archimedean in fuzzy metric space. Using the same concept for Menger PM spaces, Mishra et al. [22] proved the common fixed point theorem for six maps, Also they introduced semi-compatibility. In this paper, we generalized the theorem [22] for family of maps and proved the common fixed point theorems using the pair of semi-compatible and reciprocally continuous maps for one pair and $R$-weakly commuting maps for another pair in Menger WNAPM-spaces. Our results extends and generalizes several known results in metric spaces, probabilistic metric spaces and the similar spaces.
\end{abstract}

\section{INTRODUCTION}

In 1974, non-Archimedean probabilistic metric space and some topological preliminaries on them were first studied by Istratescu and Crivat [25] (see also [23], [24]). The existance of fixed points of mappings on non-Archimedean Menger spaces have been given by Istratescu ([26], [27]) as a result of the generalizations of some of the results of Sherwood [11] and Sehgal and Bharucha-Reid [28]. While Achari [12] studied the fixed points of quasi-contraction type mappings in non-Archimedean PM-spaces and generalized the results of [11], [26] and [28]. In 1982 Sessa [21] introduced the notion of weakly commuting maps as a generalization of commuting maps in metric spaces. In 1986, Jungck [6] introduced the concept of compatible mapping and proved some common fixed point theorems of compatible mappings in metric space. He shows that weakly commuting mappings are compatible but the converse is not true. This compatibility condition has further been weakened by introducing the notion of weakly compatible mappings by Jungck and Rhoades [5].

Received by the editors April 29, 2013. Revised August 04, 2013. Accepted August 05, 2013. 2010 Mathematics Subject Classification. 54H25, 47H10.

Key words and phrases. non-Archimedean Menger PM-spaces, weak non-Archimedean Menger PM-space, R-weakly commuting maps, semi-compatible and reciprocally continuous maps, common fixed point.

${ }^{*}$ Corresponding author. 
In 1997, Cho et al. [30] introduced the concepts of compatible maps of type $(\mathcal{A})$, in non-Archimedean Menger PM-spaces and proved some interesting results of common fixed point. In fact compatibility and compatibility of type $(\mathcal{A})$ are equivalent under some conditions.

Pathak et al. [8], [9], [10] introduced compatible maps of type $(\mathcal{B})$, type $(\mathcal{C})$ and type $(\mathcal{P})$ in metric spaces. Singh et al. [2] introduced the notion of semi-compatible maps in fuzzy metric spaces. In fact in particular, the semi-compatible maps is equivalent to the compatible maps and compatible maps of type $(\mathcal{A})$ or $(\alpha)$ and of compatible maps of type $(\mathcal{B})$ under some conditions on the maps.

Pant [17] introduced the concept of R-weakly commuting maps in metric spaces. Later on Cho et al. [30] generalized this idea and gave the concept of R-weakly commuting maps of type $A_{g}$. Vasuki [18] proved some common fixed point theorem for R-weakly commuting maps in fuzzy metric spaces. In 2009, Khan and Sumitra [13] introduced the concept of R-weakly commuting maps in non-Archimedean Menger PM-spaces and proved a common fixed point theorem for three pointwise R-weakly commuting mappings in complete non-Archimedean Menger PM-spaces. Several authors have already studied fixed point theorems in Non-Archimedean Menger PMspaces for more details, we refer the reader to Singh et al. [1], Khan and Sumitra [14], [15], Rashwan and Moustafa [16] and Singh et al. [19]

In the present paper we prove the fixed point theorem using the pair of semicompatible and reciprocally continuous maps for one pair and $R$-weakly commuting maps for another pair in Menger PM-spaces. Further we obtain a common fixed point theorems for six maps and one corollary for four maps via rational inequality. Our result generalizes and extends many results in the existing literature.

\section{Preliminaries}

Definition 2.1 ([3]). A triangular norm $\Delta$ (shortly t-norm) is a binary operation on the unit interval $[0,1]$ such that for all $a, b, c, d \in[0,1]$ and the following conditions are satisfied:

(i) $\Delta(a, 1)=a$;

(ii) $\Delta(a, b)=\Delta(b, a)$;

(iii) $\Delta(a, b) \leq \Delta(c, d)$ whenever $a \leq c$ and $b \leq d$;

(iv) $\Delta(\Delta(a, b)), c)=\Delta(a, \Delta(b, c))$.

Definition $2.2([3])$. A distribution function is a function $\mathcal{F}:(-\infty,+\infty) \rightarrow[0,1]$ 
that is if it is left continuous on $\mathbb{R}$, non-decreasing and such that $\mathcal{F}(-\infty)=0$, $\mathcal{F}(+\infty)=1$.

Let $\Delta$ be the set of all distribution functions and denoted by $\mathcal{H}(t)$ the function defined as

$$
\mathcal{H}(t)= \begin{cases}0 & \text { if } t \leq 0 \\ 1 & \text { if } t>0\end{cases}
$$

if $X$ is a non empty set, $\mathcal{F}: X \times X \rightarrow \Delta$ is called probabilistic distance on $X$ and $\mathcal{F}(x, y)$ is also usually denoted by $\mathcal{F}_{x, y}$.

Definition 2.3 ([25], [26]). An ordered pair $(X, \mathcal{F})$ is said to be non-Archimedean probabilistic metric space (shortly N.A. PM-space) if $X$ non-empty set and $\mathcal{F}$ is a probabilistic distance satisfying, for all $x, y, z \in X$ and $s, t \geq 0$, the following conditions :

(i) $F(x, y ; t)=1 \Leftrightarrow x=y$;

(ii) $F(x, y ; t)=F(y, x ; t)$;

(iii) $F(x, y ; 0)=0$;

(iv) $F(x, y ; t)=1, F(y, z ; s)=1 \Rightarrow F(x, z ; \max \{s, t\})=1$.

Remark 2.4. Every Metric space $(X, d)$ can always be realized as a PM-space by considering $\mathcal{F}: X \times X \rightarrow \Delta$ defined by $\mathcal{F}(x, y)=\mathcal{H}(t-d(x, y))$, for all $x, y \in X$ and for all $t>0$. So PM- space offer a wider framework than that of metric spaces and are general enough to cover even wider statistical situations.

The ordered triple $(X, \mathcal{F}, \Delta)$ is called a non-Archimedean Menger probabilistic metric space (shortly Menger NAPM-space) if $(X, \mathcal{F})$ is a NAPM-space, $\Delta$ is a tnorm and the following condition is also satisfied:

(v) $F(x, z ; \max \{t, s\}) \geq \Delta(\mathcal{F}(x, y ; t), \mathcal{F}(y, z ; s))$, for all $x, y, z \in X$ and $t, s>0$. Recently Mishra et al. [22] defined the WNAMP-spaces as follows. If the triangular inequality $(\mathrm{v})$ is replaced by the following:

$($ WNA $) \quad \mathcal{F}(x, z ; t) \geq \max \{\Delta(\mathcal{F}(x, y ; t), \mathcal{F}(y, z ; t / 2)), \Delta(\mathcal{F}(x, y ; t / 2), \mathcal{F}(y, z ; t))\}$, for all $x, y, z \in X$ and $t>0$,

then the triple $(X, \mathcal{F}, \Delta)$ is called a weak non-Archimedean Menger probabilistic metric space (shortly WNAPM-space). Obviously every Menger NAPM-space is itself WNA-space.

Remark 2.5. Condition (WNA) does not imply that $\mathcal{F}(y, z ; t)$ is nondecreasing and thus a Menger WNAPM-space is not necessarily a Menger PM-space. If $\mathcal{F}(y, z ; t)$ is nondecreasing, then a Menger WNA-space is a Menger PM-space. 
Definition 2.6 ([20], [30]). An N.A. Menger PM-space $(X, F, \Delta)$ is said to be of type $(C)_{g}$ if there exists a $g \in \Omega$ such that

$$
g(F(x, z ; t)) \leq g(F(x, y ; t))+g(F(y, z ; t))
$$

for all $x, y, z \in X, t \geq 0$, where $\Omega=\{g \mid g:[0,1] \rightarrow[0, \infty)$ is continuous, strictly decreasing with $g(1)=0$ and $g(0)<\infty$.

Definition 2.7 ([20], [30]). A N.A. Menger PM-space $(X, F, \Delta)$ is said to be of type $(D)_{g}$ if there exists a, $g \in \Omega$ such that $g\left(\Delta\left(t_{1}, t_{2}\right)\right) \leq g\left(t_{1}\right)+g\left(t_{2}\right)$ for all $t_{1}, t_{2} \in[0,1]$.

Remark 2.8 ([22]). If a Menger WNAPM-space is of type $(D)_{g}$, then $(X, \mathcal{F}, \Delta)$ is of type $(C)_{g}$. On the other hand, if $(X, \mathcal{F}, \Delta)$ is WNAPM-space such that $\Delta(r, s)=$ $\max (r+s-1,0)$, for all $r, s \in[0,1]$, then $(X, \mathcal{F}, \Delta)$ is of type $(D)_{g}$ for $g \in \Omega$ and $g(t)=1-t, t \geq 0$.

Throughout this paper $(X, \mathcal{F}, \Delta)$ is a complete Menger WNAPM-space with of type $(D)_{g}$ with a continuous strictly increasing t-norm $\Delta$. Let $\phi:[0,+\infty) \rightarrow[0,+\infty)$ be a function satisfying the condition:

$(\Phi) ;(\phi)$ is upper semi-continuous from the right and $\phi(t)<t$ for $t>0$.

Example 2.9. Let $X=[0, \infty), \Delta(a, b)=a b$ for every $a, b \in[0,1]$. Define $\mathcal{F}(x, y ; t)$ by: $\mathcal{F}(x, y ; 0)=0, \mathcal{F}(x, x ; t)=1$ for all $t>0, \mathcal{F}(x, y ; t)=t$ for $x \neq y$ and $0<t \leq 1$, $\mathcal{F}(x, y ; t)=t / 2$ for $x \neq y$ and $1<t \leq 2, \mathcal{F}(x, y ; t)=1$ for $x \neq y$ and $t>2$. Then $(X, \mathcal{F}, \Delta)$ is a Menger WNAPM-space, but it is not a PM-space.

Definition 2.10 ([22]). Two self maps $A$ and $B$ of a Menger WNAPM-space $(X, \mathcal{F}, \Delta)$ are said to be semi-compatible if $g\left(\mathcal{F}\left(A, B x_{n}, B z ; t\right)\right) \rightarrow 0$ for all $t>0$ whenever $\left\{x_{n}\right\}$ is a sequence in $X$ such that $A x_{n}, B x_{n} \rightarrow z$ for some $z$ in $X$ as $n \rightarrow+\infty$

Definition 2.11 ([22]). A pair of self maps $(A, B)$ of a Menger WNAPM-space $(X, \mathcal{F}, \Delta)$ are said to be reciprocally continuous if $g\left(\mathcal{F}\left(A B x_{n}, A z ; t\right)\right) \rightarrow 0$ and $g\left(\mathcal{F}\left(B A x_{n}, B z ; t\right)\right) \rightarrow 0$ for all $t>0$, whenever there exists a sequence $\left\{x_{n}\right\}$ in $X$ such that $A x_{n} \rightarrow z, B x_{n} \rightarrow z$ for some $z$ in $X$ as $n \rightarrow+\infty$

Lemma $2.12([20])$. If a function $\phi:[0,+\infty) \rightarrow[0,+\infty)$ satisfies the condition $(\Phi)$ then

(i) For all $t \geq 0, \lim _{\mathrm{n} \rightarrow \infty} \phi^{\mathrm{n}}(t)=0$, where $\phi^{\mathrm{n}}(t)$ is the $n$th iteration of $\phi(t)$.

(ii) If $\left\{t_{n}\right\}$ is a non decreasing sequence of real numbers and $t_{n+1} \leq \phi\left(t_{n}\right) n=$ $1,2, \ldots$, then $\lim _{n \rightarrow \infty} t_{n}=0$. In particular, if $t \leq \phi(t)$, for each $t \geq 0$, then $t=0$. 
Lemma $2.13([30])$. Let $\left\{y_{n}\right\}$ be a sequence in $X$ such that $\lim _{n \rightarrow \infty} F\left(y_{n}, y_{n+1} ; t\right)=$ 1 for each $t>0$. If the sequence $\left\{y_{n}\right\}$ is not a Cauchy sequence in $X$, then there exists $\in_{0}>0, t_{0}>0$, and two sequences $\left\{m_{i}\right\}$ and $\left\{n_{i}\right\}$ of positive integers such that (i) $m_{i} \geq n_{i+1}$ and $n_{i} \rightarrow \infty$ as $i \rightarrow \infty$

(ii) $F\left(y_{m i}, y_{n i} ; t_{0}\right)<1-\epsilon_{0}$ and $F\left(y_{m_{i}-1}, y_{n i} ; t_{0}\right) \geq 1-\epsilon_{0}, i=1,2, \ldots$

Proposition 2.14. Let $A$ and $B$ be two self maps of a Menger WNAPM-space $(X, \mathcal{F}, \Delta)$. The following conditions hold:

(a) if $B$ is continuous, then the pair $(A, B)$ is compatible of type $(A-1)$ if and only if $(A, B)$ is semi compatible.

(b) if $A$ is continuous, then the pair $(A, B)$ is compatible of type $(A-2)$ if and only if $(A, B)$ is semi compatible.

Proposition 2.15. Let $A$ and $B$ be two self maps of a Menger WNAPM-space $(X, \mathcal{F}, \Delta)$. Assume that $(A, B)$ is reciprocally continuous, then $(A, B)$ is semicompatible if and only if $(A, B)$ is compatible.

Proposition 2.16. Let $A$ and $B$ be two self maps of a Menger WNAPM-space $(X, \mathcal{F}, \Delta)$. If the pair $(A, B)$ is semi compatible and reciprocally continuous and $\left\{x_{n}\right\}$ is a sequence in $X$ such that $A x_{n} \rightarrow z, B x_{n} \rightarrow z$ for some $z \in X$ as $n \rightarrow+\infty$, then $A z=B z$.

We define the R-weakly commuting maps for Weak non-Archimedean Menger PM-spaces;

Definition 2.17. Two maps $A$ and $S$ of a weak non-Archimedean Menger PM space $(X, \mathcal{F}, \Delta)$ into itself are said to be $R$-weakly commuting if there exists some $R>0$ such that $g(F(A S x, S A x ; t)) \leq g(F(A S x, S A x ; t / R))$ for every $x \in X$ and $t>0$.

\section{Main Results}

We prove the following lemma:

Lemma 3.1. Let $S$ and $T$ be self mappings of a complete Menger WNAPM-space $(X, \mathcal{F}, \triangle)$ of type $(D)_{g}$, and $\left\{A_{n}\right\}_{n=1}^{\infty}$ be a family of self mappings satisfying the following conditions:

(i) $A_{i}(X) \subseteq T(X), A_{j}(X) \subseteq S(X)$;

(ii) for all $x, y \in X$ and $t>0$, 


$$
\begin{aligned}
g\left(F\left(A_{i} x, A_{j} y ; t\right)\right) \leq & \phi\left[\operatorname { m a x } \left\{g(F(S x, T y ; t)), g\left(F\left(S x, A_{i} x ; t\right)\right), g\left(F\left(T y, A_{j} y ; t\right)\right),\right.\right. \\
& \frac{1}{2}\left(g\left(F\left(S x, A_{j} y ; t\right)\right)+g\left(F\left(T y, A_{i} x ; t\right)\right)\right), \\
& \sqrt{g\left(F\left(A_{j} y, T y ; t\right)\right) \cdot g(F(T y, S x ; t))}, \\
& \left.\left.\frac{g\left(F\left(T y, A_{j} y ; t\right)\right) g\left(F\left(A_{j} y, S x ; t\right)\right)}{g(F(S x, T y ; t))}\right\}\right],
\end{aligned}
$$

where the function $\phi:[0,+\infty) \rightarrow[0,+\infty)$ satisfies the condition $(\Phi)$.

For $i=2 n-1, j=2 n,(n \in N)$ and $i \neq j$, for any $x_{0} \in X$, then the sequence $\left\{y_{n}\right\}$ defined for all $n=1,2,3, \ldots$, by

$$
y_{2 n}=A_{2 n+1}\left(x_{2 n}\right)=T x_{2 n+1}, y_{2 n-1}=A_{2 n}\left(x_{2 n-1}\right)=S x_{2 n} \quad n=1,2,3, \ldots
$$

is a Cauchy sequence in $X$ provided that $\lim _{n \rightarrow \infty} g\left(F\left(y_{n}, y_{n+1} ; t\right)\right)=0$ for all $t>0$.

Proof. In view of $(i)$ one can define the sequence $\left\{y_{n}\right\}$. Since $g \in \Omega$, it follows that $\lim _{n \rightarrow \infty} F\left(y_{n}, y_{n+1}\right)=1$ for each $t>0$ iff (if and only if ) $\lim _{n \rightarrow \infty} g\left(F\left(y_{n}, y_{n+1} ; t\right)\right)=$ 0 for each $t>0$. By Lemma 2.13 if $\left\{y_{n}\right\}$ is not a Cauchy sequence in $X$, there exist $\epsilon_{0}>0, t_{0}>0$ and two sequences $\left\{m_{i}\right\}$ and $\left\{n_{i}\right\}$ of positive integers such that:

(a) $m_{i}>n_{i}+1$ and $n_{i} \rightarrow \infty$ as $i \rightarrow \infty$

(b) $g\left(F\left(y_{m_{i}}, y_{n_{i}} ; t_{0}\right)>g\left(1-\epsilon_{0}\right)\right.$ and $g\left(F\left(y_{m_{i}-1}, y_{n_{i}} ; t_{0}\right) \leq\left(1-\epsilon_{0}\right) i=1,2,3, \ldots\right.$ Since $g(t)=1-t$, we have

$$
\begin{aligned}
g\left(1-\epsilon_{0}\right)<g\left(F\left(y_{m_{i}}, y_{n_{i}} ; t_{0}\right)\right) & \leq g\left(F\left(y_{m_{i}}, y_{m_{i}-1} ; t_{0}\right)\right)+g\left(F\left(y_{m_{i}-1}, y_{n_{i}} ; t_{0}\right)\right) \\
& \leq g\left(F\left(y_{m_{i}}, y_{m_{i}-1} ; t_{o}\right)\right)+g\left(1-\epsilon_{0}\right)
\end{aligned}
$$

As $i \rightarrow \infty$, we have

$$
\lim _{i \rightarrow \infty} g\left(F\left(y_{m_{i}}, y_{n_{i}} ; t_{0}\right)\right)=g\left(1-\epsilon_{0}\right)
$$

on the other hand, we have

$$
g\left(1-\epsilon_{0}\right)<g\left(F\left(y_{m_{i}}, y_{n_{i}} ; t_{0}\right)\right) \leq g\left(F\left(y_{n_{i}}, y_{n_{i}+1} ; t_{0}\right)\right)+g\left(F\left(y_{m_{i}}, y_{n_{i}+1} ; t_{0}\right)\right) .
$$

Now consider $g\left(F\left(y_{m_{i}}, y_{n_{i}+1} ; t_{0}\right)\right.$ and assume that both $m_{i}$ and $n_{i}$ are even. Then by $(i i)$ of Lemma 3.1 , we have

$$
\begin{aligned}
g\left(F\left(y_{m_{i}}, y_{n_{i}+1} ; t_{0}\right)\right) & =g\left(F\left(A_{2 n+1}\left(x_{m i}\right), A_{2 n}\left(x_{n_{i}+1}\right) ; t_{0}\right)\right) \\
& \leq \phi\left[\operatorname { m a x } \left\{g\left(F\left(S x_{m_{i}}, T x_{n_{i}+1} ; t_{0}\right)\right), g\left(F\left(S x_{m_{i}}, A_{2 n+1}\left(x_{m_{i}}\right) ; t_{0}\right)\right),\right.\right.
\end{aligned}
$$




$$
\begin{aligned}
& g\left(F\left(T x_{n_{i}+1}, A_{2_{n}}\left(x_{n_{i}+1}\right) ; t_{0}\right)\right) \\
& \frac{1}{2}\left(g\left(F\left(S x_{m_{i}}, A_{2 n}\left(x_{n_{i}+1}\right) ; t_{0}\right)\right)+g\left(F\left(T x_{n_{i}+1}, A_{2 n+1}\left(x_{m_{i}}\right) ; t_{0}\right)\right)\right), \\
& \sqrt{g\left(F\left(A_{2 n}\left(x_{n_{i}+1}\right), T x_{n_{i}+1} ; t_{0}\right)\right) \cdot g\left(F\left(T x_{n_{i}+1}, S x_{m_{i}} ; t_{0}\right)\right)}, \\
& \left.\left.\frac{g\left(F\left(T x_{n_{i}+1}, A_{2 n}\left(x_{n_{i}+1}\right) ; t_{0}\right)\right) \cdot g\left(F\left(A_{2 n}\left(x_{n_{i}+1}\right), S x_{m_{i}} ; t_{0}\right)\right)}{g\left(F\left(S x_{m_{i}}, T x_{n_{i}+1} ; t_{0}\right)\right)}\right\}\right] \\
\leq & \phi\left[\operatorname { m a x } \left\{g\left(F\left(y_{m_{i}-1}, y_{n_{i}} ; t_{0}\right)\right), g\left(F\left(y_{m_{i}-1}, y_{m_{i}} ; t_{o}\right)\right),\right.\right. \\
& g\left(f\left(y_{n_{i}}, y_{n_{i}+1} ; t_{0}\right)\right), \\
& \frac{1}{2}\left(g\left(F\left(y_{m_{i}-1}, y_{n_{i}+1} ; t_{0}\right)\right)+g\left(F\left(y_{n_{i}}, y_{m_{i}} ; t_{0}\right)\right)\right) \\
& \sqrt{g\left(F\left(y_{n_{i}+1}, y_{n_{i}} ; t_{0}\right)\right), g\left(F\left(y_{n_{i}}, y_{m_{i}-1} ; t_{o}\right)\right)}, \\
& \left.\left.\frac{g\left(F\left(y_{n_{i}}, y_{n_{i}+1} ; t_{0}\right)\right) \cdot g\left(F\left(y_{n_{i}+1}, y_{m_{i}-1} ; t_{o}\right)\right)}{g\left(F\left(y_{m_{i}-1}, y_{n_{i}} ; t_{0}\right)\right)}\right\}\right]
\end{aligned}
$$

that is

$$
\begin{aligned}
& g\left(F\left(y_{m_{i}}, y_{n_{i}+1} ; t_{0}\right)\right) \leq \phi\left[\operatorname { m a x } \left\{g\left(1-\epsilon_{0}\right), g\left(F\left(y_{m_{i}-1}, y_{m_{i}} ; t_{o}\right)\right),\right.\right. \\
& g\left(f\left(y_{n_{i}}, y_{n_{i}+1} ; t_{0}\right)\right), \\
& \frac{1}{2}\left\{\left(g\left(1-\epsilon_{0}\right)+g\left(F\left(y_{n_{i}}, y_{n_{i}+1} ; t_{0}\right)\right)\right)+g\left(F\left(y_{n_{i}}, y_{m_{i}} ; t_{0}\right)\right)\right\}, \\
& \sqrt{g\left(F\left(y_{n_{i}+1}, y_{n_{i}} ; t_{0}\right)\right) \cdot g\left(1-\epsilon_{0}\right)}, \\
&\left.\left.\frac{g\left(F\left(y_{n_{i}}, y_{n_{i}+1} ; t_{0}\right)\right) \cdot\left(g\left(F\left(y_{n_{i}+1}, y_{n_{i}} ; t_{o}\right)\right)+g\left(F\left(y_{n_{i}}, y_{m_{i}-1} ; t_{o}\right)\right)\right)}{g\left(1-\epsilon_{0}\right)}\right\}\right]
\end{aligned}
$$

Putting this value in (3.3), using (3.2) and taking $i \rightarrow \infty$,

$$
\begin{aligned}
g\left(1-\epsilon_{0}\right) & \leq \phi\left[\operatorname{Max}\left\{g\left(1-\epsilon_{0}\right), 0,0, g\left(1-\epsilon_{0}\right), 0,0\right\}\right] \\
& \leq \phi g\left(1-\epsilon_{0}\right)<g\left(1-\epsilon_{0}\right),
\end{aligned}
$$

which is a contradiction. Hence the sequence $\left\{y_{n}\right\}$ is a Cauchy sequence in $X$.

Theorem 3.2. Let $S$ and $T$ be self mappings of a complete Menger WNAPM-space $(X, \mathcal{F}, \triangle)$ of type $(D)_{g}$, and $\left\{A_{n}\right\}_{n=1}^{\infty}$ be a family of self mappings satisfying (i) and (ii) of Lemma 3.1 and suppose that:

(iii) the pair $\left\{A_{i}, S\right\}$ is reciprocally continuous and semi-compatible and $\left\{A_{j}, T\right\}$ is $R$-weakly commuting.

Then the mapping $\left\{A_{n}\right\}, S$ and $T$ have a unique common fixed point.

Proof. Since $A_{i}(X) \subseteq T(X)$, for any $x_{0} \in X$, there exists a point $x_{1} \in X$ such that $A_{1}\left(x_{0}\right)=T x_{1}$. Since $A_{j}(X) \subseteq S(X)$, for this $x_{1}$ we can choose a point $x_{2} \in X$ such 
that $A_{2}\left(x_{1}\right)=S x_{2}$ and so on. Inductively, We can define a sequence $\left\{y_{n}\right\}$ in $X$, such that

$$
y_{2 n}=A_{2 n+1}\left(x_{2 n}\right)=T x_{2 n+1}, \quad y_{2 n-1}=A_{2 n}\left(x_{2 n-1}\right)=S x_{2 n} \quad n=1,2,3 \ldots
$$

If we prove that, for all $t>0, g\left(F\left(A_{2 n+1}\left(x_{n}\right), A_{2 n}\left(x_{n-1}\right) ; t\right)\right)=0$ then by Lemma 3.1 , we can conclude that the sequence $\left\{y_{n}\right\}$ is a Cauchy sequence in $X$, for this taking (ii) of Lemma 3.1, we have

$$
\begin{aligned}
& g\left(F\left(y_{2 n}, y_{2 n-1} ; t\right)\right)= g\left(F\left(A_{2 n+1}\left(x_{2 n}\right), A_{2 n}\left(x_{2 n-1}\right) ; t\right)\right) \\
& \leq \phi\left[\operatorname { m a x } \left\{g\left(F\left(S x_{2 n}, T x_{2 n-1} ; t\right)\right), g\left(F\left(S x_{2 n}, A_{2 n+1}\left(x_{2 n}\right) ; t\right)\right),\right.\right. \\
& g\left(F\left(T x_{2 n-1}, A_{2 n}\left(x_{2 n-1}\right) ; t\right)\right), \\
& \frac{1}{2}\left(g\left(F\left(S x_{2 n}, A_{2 n}\left(x_{2 n-1}\right) ; t\right)\right)+g\left(F\left(T x_{2 n-1}, A_{2 n+1}\left(x_{2 n}\right) ; t\right)\right)\right), \\
& \sqrt{g\left(F\left(A_{2 n}\left(x_{2 n-1}\right), T x_{2 n-1} ; t\right)\right) \cdot g\left(F\left(T x_{2 n-1}, S x_{2 n}\right) ; t\right)}, \\
&\left.\frac{g\left(F\left(T x_{2 n-1}, A_{2 n}\left(x_{2 n-1}\right) ; t\right)\right) g\left(F\left(A_{2 n}\left(x_{2 n-1}\right), S x_{2 n} ; t\right)\right)}{g\left(F\left(S x_{2 n}, T x_{2 n-1} ; t\right)\right)}\right\} \\
&= \phi\left[\operatorname { m a x } \left\{g\left(F\left(y_{2 n-1}, y_{2 n-2} ; t\right)\right), g\left(F\left(y_{2 n-1}, y_{2 n} ; t\right)\right),\right.\right. \\
& g\left(F\left(y_{2 n-2}, y_{2 n-1} ; t\right)\right), \frac{1}{2}\left(g\left(F\left(y_{2 n-1}, y_{2 n-1} ; t\right)\right)+g\left(F\left(y_{2 n-2}, y_{2 n} ; t\right)\right)\right), \sqrt{g\left(F\left(y_{2 n-1}, y_{2 n-2} ; t\right)\right) \cdot g\left(F\left(y_{2 n-2}, y_{2 n-1} ; t\right)\right)}, \\
&\left.\frac{g\left(F\left(y_{2 n-2}, y_{2 n-1} ; t\right)\right) \cdot g\left(F\left(y_{2 n-1}, y_{2 n-1} ; t\right)\right)}{g\left(F\left(y_{2 n-1}, y_{2 n-2} ; t\right)\right)}\right\} \\
& g\left(F\left(y_{2 n}, y_{2 n-1} ; t\right)\right) \leq \phi\left[\operatorname { m a x } \left\{g\left(F\left(y_{2 n-1}, y_{2 n-2} ; t\right)\right), g\left(F\left(y_{2 n-1}, y_{2 n} ; t\right)\right),\right.\right. \\
& g\left(F\left(y_{2 n-2}, y_{2 n-1} ; t\right)\right), \frac{1}{2}\left(g\left(F\left(y_{2 n-2}, y_{2 n-1} ; t\right)\right)+g\left(F\left(y_{2 n-1}, y_{2} ; t\right)\right)\right), \\
&\left.\left.\sqrt{\left(g\left(F\left(y_{2 n-1}, y_{2 n-2} ; t\right)\right)\right)^{2}} \frac{g\left(F\left(y_{2 n-1}, y_{2 n-2} ; t\right)\right) g(1)}{g\left(F\left(y_{2 n-1}, y_{2 n-2} ; t\right)\right)}\right\}\right]
\end{aligned}
$$

Case I : If $g\left(F\left(y_{2 n}, y_{2 n-1} ; t\right)\right) \geq g\left(F\left(y_{2 n-1}, y_{2 n-2} ; t\right)\right)$, then $g\left(F\left(y_{2 n}, y_{2 n-1} ; t\right)\right) \leq \phi g\left(F\left(y_{2 n}, y_{2 n-1} ; t\right)\right)$ which is a contradiction.

Case II : If $g\left(F\left(y_{2 n}, y_{2 n-1} ; t\right)\right) \leq g\left(F\left(y_{2 n-1}, y_{2 n-2} ; t\right)\right)$,

then get $g\left(F\left(y_{2 n}, y_{2 n-1} ; t\right)\right) \leq \phi g\left(F\left(y_{2 n-1}, y_{2 n-2} ; t\right)\right)$.

Similarly we can obtain $g\left(F\left(y_{2 n+1}, y_{2 n} ; t\right)\right) \leq \phi g\left(F\left(y_{2 n}, y_{2 n-1} ; t\right)\right)$ for all $t>0$ and $n=1,2,3, \ldots$, thus we get $g\left(F\left(y_{n}, y_{n+1} ; t\right)\right) \leq \phi g\left(F\left(y_{n-1}, y_{n} ; t\right)\right)$,

$\lim _{n \rightarrow \infty} g\left(F\left(A_{2 n+1} x_{n}, A_{2 n}\left(x_{n+1}\right) ; t\right)\right)=0, \quad \lim _{n \rightarrow \infty} g\left(F\left(y_{2 n}, y_{2 n-1} ; t\right)\right)=0$ for all $t>0$ which implies that $\left\{y_{n}\right\}$ is a Cauchy sequence in $X$ by Lemma 3.1. Since $X$ is 
complete, then the sequence $\left\{y_{n}\right\}$ converges to a point $z$ in $X$ and so the subsequences $\lim _{n \rightarrow \infty} A_{2 n+1}\left(x_{2 n}\right), \lim _{n \rightarrow \infty} A_{2 n}\left(x_{2 n+1}\right), \lim _{n \rightarrow \infty} S x_{2 n}$ and $\lim _{x \rightarrow \infty} T x_{2 n+1}$ of seq. $\left\{y_{n}\right\}$ also converge to the limit $z$.

Since the pair $\left(A_{i}, S\right)$ is reciprocally continuous,

$$
\lim _{n \rightarrow \infty} g\left(F\left(A_{i} S x_{2 n}, A_{i} z ; t\right)\right) \rightarrow 0 \text { and } g\left(F\left(S A_{i} x_{2 n}, S z ; t\right)\right) \text { as } n \rightarrow \infty
$$

By semi compatibility of $\left(A_{i}, S\right)$ we get $g\left(F\left(A_{i} S x_{2 n}, A_{i} z ; t\right)\right) \rightarrow 0$ that is $A_{i} z=S z$. Now taking condition ( $i i)$ of Lemma 3.1 with $x=z$ and $y=x_{2 n+1}$

$$
\begin{aligned}
g\left(F\left(A_{i} z, A_{j} x_{2 n+1} ; t\right)\right) \leq & \phi\left[\operatorname { m a x } \left\{g\left(F\left(S z, T x_{2 n+1} ; t\right)\right), g\left(F\left(S z, A_{i} z ; t\right)\right),\right.\right. \\
& g\left(F\left(T x_{2 n+1}, A_{j} x_{2 n+1} ; t\right)\right), \\
& \frac{1}{2}\left(g\left(F\left(S z, A_{j} x_{2 n+1} ; t\right)\right)+g\left(F\left(T x_{2 n+1}, A_{i} z ; t\right)\right)\right), \\
& \sqrt{g\left(F\left(A_{j}\left(T x_{2 n+1}\right), T x_{2 n+1} ; t\right)\right) \cdot g\left(F\left(T x_{2 n+1}, S z ; t\right)\right),} \\
& \left.\left.\frac{g\left(F\left(A_{j}\left(x_{2 n+1}\right), T x_{2 n+1} ; t\right)\right) . g\left(F\left(S z, A_{j} x_{2 n+1} ; t\right)\right)}{g\left(F\left(S z, T x_{2 n+1} ; t\right)\right)}\right\}\right]
\end{aligned}
$$

which on letting limit $n \rightarrow \infty$

$$
\begin{aligned}
g\left(\left(A_{i} z, z ; t\right)\right) \leq & \phi[\max \{g(F(S z, z ; t)), g(F(S z, S z ; t)),, g(F(z, z ; t)), \\
& \frac{1}{2}\left\{g\left(F\left(A_{i} z, z ; t\right)\right)+g\left(F\left(z, A_{i} z ; t\right)\right)\right\}, \sqrt{g\left(F\left(z, A_{i} z ; t\right)\right) \cdot g(F(z, z ; t)),} \\
& \left.\left.\frac{g(F(z, z ; t)) \cdot g\left(F\left(A_{i} z, T z ; t\right)\right)}{g\left(F\left(A_{i} z, z ; t\right)\right)}\right\}\right] \\
g\left(\left(A_{i} z, z ; t\right)\right) \leq \phi[ & \left.M a x\left\{g\left(F\left(A_{i} z, z ; t\right)\right), 0,0, g\left(F\left(A_{i} z, z ; t\right)\right), 0,0\right\}\right]
\end{aligned}
$$

i.e. $A_{i} z=z$ hence $A_{i} z=S z=z$.

Since the pair $\left(A_{j}, T\right)$ is $R$-weakly commuting, so

$$
g\left(F\left(A_{j} T x_{2 n+1}, T A_{j} x_{2 n+1} ; t\right)\right) \leq g\left(F\left(A_{j} x_{2 n+1}, T x_{2 n+1} ; t / R\right)\right)
$$

which gives

$$
\lim _{n \rightarrow \infty} A_{j} T x_{2 n+1}=\lim _{n \rightarrow \infty} T A_{j} x_{2 n+1}=T z
$$

(as $T$ is continuous).

We have to show that $T z=z$. To do this contrary suppose that $T z \neq z$. Then by (ii) of Lemma 3.1 


$$
\begin{aligned}
g\left(F\left(A_{i} z, A_{j} T x_{2 n} ; t\right)\right) \leq & \phi\left[\operatorname { m a x } \left\{g\left(F\left(S z, T T x_{2 n} ; t\right)\right), g\left(F\left(S z, A_{i} z ; t\right)\right),\right.\right. \\
& g\left(F\left(T T x_{2 n}, A_{j}\left(T x_{2 n}\right) ; t\right)\right), \\
& \frac{1}{2}\left(g\left(F\left(S z, A_{j} T x_{2 n} ; t\right)\right)+g\left(F\left(T T x_{2 n}, A_{i} z ; t\right)\right)\right), \\
& \sqrt{g\left(F\left(A_{j}\left(T x_{2 n}\right), T T x_{2 n} ; t\right)\right) \cdot g\left(F\left(T T x_{2 n}, S z ; t\right)\right),} \\
& \left.\left.\frac{g\left(F\left(A_{j}\left(T x_{2 n}\right), T T x_{2 n} ; t\right)\right) \cdot g\left(F\left(S z, A_{j} T x_{2 n} ; t\right)\right)}{g\left(F\left(S z, T T x_{2 n} ; t\right)\right)}\right\}\right]
\end{aligned}
$$

which on letting limit $n \rightarrow \infty$

$$
\begin{aligned}
g((z, T z ; t)) \leq & \phi[\max \{g(F(z, T z ; t)), g(F(z, z ; t)), g(F(T z, T z ; t)), \\
& \frac{1}{2}\{g(F(z, T z ; t))+g(F(T z, z ; t))\}, \sqrt{(g(F(T z, T z ; t)) \cdot g(F(z, T z ; t))}, \\
& \left.\left.\frac{g(F(T z, T z ; t)) . g(F(z, T z ; t))}{g(F(z, T z ; t))}\right\}\right]
\end{aligned}
$$

i.e., $g(F(z, T z ; t)) \leq \phi g(F(z, T z ; t))<g(F(z, T z ; t))$, which is a contradiction. Thus $z$ is a fixed point of $T$. Similarly we can show that $z$ is a fixed point of $A_{j}$. Hence $A_{i} z=A_{j} z=S z=T z=z$. Thus $z$ is a common fixed point of $A_{i}, A_{j}, S$ and $T$. The uniqueness of the common fixed point follows from inequality (ii) of Lemma 3.1 .

Corollary 3.3. Let $A_{1}, A_{2}, S$ and $T$ be four self mappings of a complete Menger $W N A P M$-spaces $(X, \mathcal{F}, \triangle)$, of type $(D)_{g}$, and satisfying:

(i) $A_{1}(X) \subseteq T(X), A_{2}(X) \subseteq S(X)$ :

(ii) the pair $\left\{A_{1}, S\right\}$ is reciprocallay continuous and semi-compatible and $\left\{A_{2}, T\right\}$ is R-weakly commuting,

(iii) for all $x, y \in X$ and $t>0$,

$$
\begin{aligned}
g\left(F\left(A_{1}(x), A_{2}(y) ; t\right)\right) \leq & \phi\left[\operatorname { m a x } \left\{g(F(S x, T y ; t)), g\left(F\left(S x, A_{1} x ; t\right)\right), g\left(F\left(T y, A_{2} y ; t\right)\right)\right.\right. \\
& \frac{1}{2}\left(g\left(F\left(S x, A_{2} y ; t\right)\right)+g\left(F\left(T y, A_{1} x ; t\right)\right)\right), \\
& \sqrt{g\left(F\left(A_{2} y, S y ; t\right)\right) \cdot g\left(F\left(A_{2} y, T y ; t\right)\right)}, \\
& \left.\left.\frac{g\left(F\left(A_{2} y, T x ; t\right)\right) \cdot g\left(F\left(A_{2} y, T y ; t\right)\right)}{g(F(S x, T y ; t))}\right\}\right] .
\end{aligned}
$$

Then $A_{1}, A_{2}, S$ and $T$ have a unique common fixed point in $X$.

Lemma 3.4. Let $P, Q, A, B, S$ and $T$ be self mappings of a complete Menger WNAPMspaces $(X, \mathcal{F}, \triangle)$, of type $(D)_{g}$, and satisfying; 
(i) $P(X) \subseteq S T(X), Q(X) \subseteq A B(X)$;

(ii) for all $x, y \in X$ and $t>0$,

$g(F(P x, Q y ; t)) \leq \phi[\operatorname{Max}\{g(F(Q y, S T y ; t)), g(F(P x, A B x ; t)), g(F(A B x, S T y ; t))$

$$
\begin{aligned}
& \frac{1}{2}(g(F(A B x, Q y ; t))+g(F(P x, S T y ; t))), \\
& \sqrt{g(F(A B x, S T y ; t)) \cdot g(F(S T y, P x ; t))}, \\
& \left.\left.\frac{g(F(A B x, P x ; t)) \cdot g(F(P x, S T y ; t))}{g(F(A B x, S T y ; t))}\right\}\right]
\end{aligned}
$$

where the function $\phi:[0,+\infty) \rightarrow[0,+\infty)$ satisfies the condition $(\Phi)$.

For any $x_{0} \in X$, then the sequence $\left\{y_{n}\right\}$ defined for all $n=0,1,2, \ldots$, by

$$
P x_{2 n}=S T\left(x_{2 n+1}\right)=y_{n}, \quad Q x_{2 n+1}=A B_{2 n+2}=y_{n+1} \quad n=0,1,2, \ldots
$$

is a Cauchy sequence in $X$ provided that $\lim _{n \rightarrow \infty} g\left(F\left(y_{n}, y_{n+1} ; t\right)\right)=0$ for all $t>0$.

Proof. In view of $(i)$ of Lemma 3.4 one can define the sequence $\left\{y_{n}\right\}$. Since $g \in \Omega$, it follows that

$\lim _{n \rightarrow \infty} F\left(y_{n}, y_{n+1}\right)=1$ for each $t>0$ if and only if $\lim _{n \rightarrow \infty} g\left(F\left(y_{n}, y_{n+1} ; t\right)\right)=0$ for each $t>0$. By Lemma 2.13, if $\left\{y_{n}\right\}$ is not a Cauchy sequence in $X$, there exist $\epsilon_{0}>0, t_{0}>0$ and two sequences $\left\{m_{i}\right\}$ and $\left\{n_{i}\right\}$ of positive integers such that:

(a) $m_{i}>n_{i}+1$ and $n_{i} \rightarrow \infty$ as $i \rightarrow \infty$

(b) $g\left(F\left(y_{m_{i}}, y_{n_{i}} ; t_{0}\right)>g\left(1-\epsilon_{0}\right)\right.$ and $g\left(F\left(y_{m_{i}-1}, y_{n_{i}} ; t_{0}\right) \leq\left(1-\epsilon_{0}\right) i=1,2, \ldots\right.$

Since $g(t)=1-t$, we have

$$
\begin{aligned}
g\left(1-\epsilon_{0}\right)<g\left(F\left(y_{m_{i}}, y_{n_{i}} ; t_{0}\right)\right) & \leq g\left(F\left(y_{m_{i}}, y_{m_{i}-1} ; t_{0}\right)\right)+g\left(F\left(y_{m_{i}-1}, y_{n_{i}} ; t_{0}\right)\right) \\
& \leq g\left(F\left(y_{m_{i}}, y_{m_{i}-1} ; t_{o}\right)\right)+g\left(1-\epsilon_{0}\right) .
\end{aligned}
$$

As $i \rightarrow \infty$, we have

$$
\lim _{i \rightarrow \infty} g\left(F\left(y_{m_{i}}, y_{n_{i}} ; t_{0}\right)\right)=g\left(1-\epsilon_{0}\right) .
$$

On the other hand, we have

$$
g\left(1-\epsilon_{0}\right)<g\left(F\left(y_{m_{i}}, y_{n_{i}} ; t_{0}\right)\right) \leq g\left(F\left(y_{n_{i}}, y_{n_{i}+1} ; t_{0}\right)\right)+g\left(F\left(y_{m_{i}}, y_{n_{i}+1} ; t_{0}\right)\right) .
$$

Now consider $g\left(F\left(y_{m_{i}}, y_{n_{i}+1} ; t_{0}\right)\right.$ and assume that both $m_{i}$ and $n_{i}$ are even. Then by $(i i)$ of Lemma 3.4, we have 


$$
\begin{aligned}
g\left(F\left(y_{m_{i}}, y_{n_{i}+1} ; t_{0}\right)\right)= & g\left(F\left(P\left(x_{m i}\right), Q\left(x_{n_{i}+1}\right) ; t_{0}\right)\right) \\
\leq & \phi\left[\operatorname { m a x } \left\{g\left(F\left(Q x_{m_{i}}, S T x_{n_{i}+1} ; t_{0}\right)\right), g\left(F\left(P x_{m_{i}}, A B x_{m_{i}} ; t_{0}\right)\right),\right.\right. \\
& g\left(F\left(A B x_{m_{i}}, S T x_{n_{i}+1} ; t_{0}\right)\right), \\
& \frac{1}{2}\left(g\left(F\left(A B x_{m_{i}}, S T x_{n_{i}+1} ; t_{0}\right)\right)+g\left(F\left(S T x_{n_{i}+1}, P x_{m_{i}}\right) ; t_{0}\right)\right), \\
& \sqrt{g\left(F\left(A B x_{m_{i}}, S T x_{n_{i}+1} ; t_{0}\right)\right) \cdot g\left(F\left(S T x_{n_{i}+1}, P x_{m_{i}} ; t_{0}\right)\right),} \\
& \left.\left.\frac{g\left(F\left(A B x_{m_{i}}, P x_{m_{i}} ; t_{0}\right)\right) \cdot g\left(F\left(P x_{m_{i}+1}, S T x_{n_{i}+1} ; t_{0}\right)\right)}{g\left(F\left(A B x_{m_{i}}, S T x_{n_{i}+1} ; t_{0}\right)\right)}\right\}\right] \\
\leq & \phi\left[\operatorname { m a x } \left\{g\left(F\left(y_{n_{i}+1}, y_{n_{i}} ; t_{0}\right)\right), g\left(F\left(y_{m_{i}}, y_{m_{i}-1} ; t_{o}\right)\right),\right.\right. \\
& g\left(f\left(y_{m_{i}-1}, y_{n_{i}} ; t_{0}\right)\right), \\
& \frac{1}{2}\left(g\left(F\left(y_{m_{i}-1}, y_{n_{i}+1} ; t_{0}\right)\right)+g\left(F\left(y_{n_{i}}, y_{m_{i}} ; t_{0}\right)\right)\right), \\
& \sqrt{g\left(F\left(y_{m_{i}-1}, y_{n_{i}} ; t_{0}\right)\right) \cdot g\left(F\left(y_{n_{i}}, y_{m_{i}} ; t_{o}\right)\right)}, \\
& \left.\left.\frac{g\left(F\left(y_{m_{i}}, y_{m_{i}-1} ; t_{0}\right)\right) \cdot g\left(F\left(y_{n_{i}}, y_{m_{i}} ; t_{o}\right)\right)}{g\left(F\left(y_{m_{i}-1}, y_{n_{i}} ; t_{0}\right)\right)}\right\}\right]
\end{aligned}
$$

that is

$$
\begin{aligned}
g\left(F\left(y_{m_{i}}, y_{n_{i}+1} ; t_{0}\right)\right) \leq & \phi\left[\operatorname { m a x } \left\{g\left(F\left(y_{n_{i}+1}, y_{n_{i}} ; t_{0}\right)\right), g\left(F\left(y_{m_{i}}, y_{m_{i}-1} ; t_{o}\right)\right), g\left(1-\epsilon_{0}\right),\right.\right. \\
\frac{1}{2}( & \left.g\left(F\left(y_{m_{i}-1}, y_{n_{i}} ; t_{0}\right)\right)+g\left(F\left(y_{n_{i}}, y_{n_{i}+1} ; t_{0}\right)\right)+g\left(F\left(y_{n_{i}}, y_{m_{i}} ; t_{0}\right)\right)\right), \\
& \sqrt{g\left(1-\epsilon_{0}\right) g\left(1-\epsilon_{0}\right)}, \\
& \left.\left.\frac{g\left(F\left(y_{m_{i}}, y_{m_{i}-1} ; t_{0}\right)\right) \cdot g\left(1-\epsilon_{0}\right)}{g\left(1-\epsilon_{0}\right)}\right\}\right] .
\end{aligned}
$$

Putting this value in (3.9), using (3.8) and taking $i \rightarrow \infty$,

$$
\begin{aligned}
g\left(1-\epsilon_{0}\right) & \leq \phi\left[\operatorname{Max}\left\{g\left(1-\epsilon_{0}\right), 0,0, g\left(1-\epsilon_{0}\right), 0,0\right\}+0\right] \\
& \leq \phi g\left(1-\epsilon_{0}\right)<g\left(1-\epsilon_{0}\right),
\end{aligned}
$$

which is a contradiction. Hence the sequence $\left\{y_{n}\right\}$ is a cauchy sequence in $X$.

Theorem 3.5. Let $P, Q, A, B, S$ and $T$ be self mappings of a complete Menger WNAPM-spaces $(X, \mathcal{F}, \triangle)$, of type $(D)_{g}$, and satisfying $(i)$ and $(i i)$ of Lemma 3.4 and suppose that:

(iii) $A B=B A, S T=T S, P B=B P, Q T=T Q$;

(iv) the pair $\{P, A B\}$ is reciprocally continuous and semi-compatible and $\{Q, S T\}$ is $R$-weakly commuting.

Then $P, Q, A, B, S$ and $T$ have a unique common fixed point in $X$. 
Proof. Since $P(X) \subseteq S T(X)$ for any $x_{0} \in X$, there exists a point $x_{1} \in X$ such that $P x_{1}=S T x_{0}=y_{0}$, and since $Q(X) \subseteq A B(X)$, for this $x_{1}$ we can choose a point $x_{2} \in X$ such that $Q x_{1}=A B x_{2}=y_{1}$. Inductively, We can construct the sequences $\left\{y_{n}\right\}$ in $X$, such that

$$
P x_{2 n}=S T\left(x_{2 n+1}\right)=y_{2 n}, \quad Q x_{2 n+1}=A B_{2 n+2}=y_{2 n+1} \quad n=0,1,2, \ldots
$$

Now since $P x_{2 n}=S T\left(x_{2 n+1}\right)$, if we prove that, for all $t>0, \lim _{n \rightarrow \infty} g\left(F\left(y_{n}, y_{n+1} ; t\right)\right)$ $=0$ then by Lemma 3.4, we can conclude that the sequence $\left\{y_{n}\right\}$ is a Cauchy sequence in $X$, for this taking ( $i i)$ of Lemma 3.4, we have

$$
\begin{aligned}
g\left(F\left(y_{2 n}, y_{2 n+1} ; t\right)\right)= & g\left(F\left(P x_{2 n}, Q x_{2 n+1} ; t\right)\right) \\
\leq & \phi\left[M a x \left\{g\left(F\left(Q x_{2 n+1}, S T x_{2 n+1} ; t\right)\right), g\left(F\left(P x_{2 n}, A B x_{2 n} ; t\right)\right),\right.\right. \\
& g\left(F\left(A B x_{2 n}, S T x_{2 n+1} ; t\right)\right), \\
& \frac{1}{2}\left(g\left(F\left(A B x_{2 n}, Q x_{2 n+1} ; t\right)\right)+g\left(F\left(P x_{2 n}, S T x_{2 n+1} ; t\right)\right)\right), \\
& \sqrt{g\left(F\left(A B x_{2 n}, S T x_{2 n+1} ; t\right)\right) \cdot g\left(F\left(S T x_{2 n+1}, P x_{2 n} ; t\right)\right)}, \\
& \left.\left.\frac{g\left(F\left(A B x_{2 n}, P x_{2 n} ; t\right)\right) \cdot g\left(F\left(P x_{2 n}, S T x_{2 n+1} ; t\right)\right)}{g\left(F\left(A B x_{2 n}, S T x_{2 n+1} ; t\right)\right)}\right\}\right] \\
g\left(F\left(y_{2 n}, y_{2 n+1} ; t\right)\right) \leq \phi[ & \max \left\{g\left(F\left(y_{2 n+1}, y_{2 n} ; t\right)\right), g\left(F\left(y_{2 n}, y_{2 n-1} ; t\right)\right),\right. \\
g\left(F\left(y_{2 n-1}, y_{2 n+1} ; t\right)\right), \frac{1}{2}\left(g\left(F\left(y_{2 n-1}, y_{2 n+1} ; t\right)\right)+g\left(F\left(y_{2 n}, y_{2 n} ; t\right)\right)\right), & \sqrt{g\left(F\left(y_{2 n-1}, y_{2 n} ; t\right)\right) \cdot g\left(F\left(y_{2 n}, y_{2 n} ; t\right)\right)}, \\
& \left.\frac{g\left(F\left(y_{2 n}, y_{2 n-1} ; t\right)\right) \cdot g\left(F\left(y_{2 n}, y_{2 n} ; t\right)\right)}{g\left(F\left(y_{2 n-1}, y_{2 n} ; t\right)\right)}\right\} .
\end{aligned}
$$

Case I : If $g\left(F\left(y_{2 n-1}, y_{2 n} ; t\right)\right) \leq g\left(F\left(y_{2 n}, y_{2 n+1} ; t\right)\right)$, then $g\left(F\left(y_{2 n}, y_{2 n+1} ; t\right)\right) \leq \phi g\left(F\left(y_{2 n}, y_{2 n+1} ; t\right)\right)$, which is a contradiction.

Case II : If $g\left(F\left(y_{2 n-1}, y_{2 n} ; t\right)\right) \geq g\left(F\left(y_{2 n-1}, y_{2 n} ; t\right)\right)$,

then we get $g\left(F\left(y_{2 n}, y_{2 n+1} ; t\right)\right) \leq \phi g\left(F\left(y_{2 n}, y_{2 n-1} ; t\right)\right)$.

Similarly we obtain that $g\left(F\left(y_{2 n+1}, y_{2 n+2} ; t\right)\right) \leq \phi g\left(F\left(y_{2 n}, y_{2 n+1} ; t\right)\right)$ for all $t>0$ and $n=0,1,2, \ldots$, thus we get $g\left(F\left(y_{n}, y_{n+1} ; t\right)\right) \leq \phi g\left(F\left(y_{n}, y_{n-1} ; t\right)\right)$ for all $t>0$ and $n=0,1,2, \ldots$ which implies that $\left\{y_{n}\right\}$ is a Cauchy sequence in $X$ by Lemma 3.4. Since $X$ is complete, then the sequence $\left\{y_{n}\right\}$ converges to a point $z$ in $X$ also, its subsequences

$\lim _{n \rightarrow \infty} P x_{2 n}, \lim _{n \rightarrow \infty} A B x_{2 n}, \lim _{n \rightarrow \infty} Q x_{2 n+1}$ and $\lim _{x \rightarrow \infty} S T x_{2 n+1}$ of seq. $\left\{y_{n}\right\}$ also converge to the limit $z$. 
Now since the pair $(P, A B)$ is reciprocally continuous, therefore we have

$$
g\left(F\left(P A B x_{2 n}, P z ; t\right)\right) \rightarrow 0 \text { and } g\left(F\left(A B P x_{2 n}, A B z ; t\right)\right) \text { as } n \rightarrow \infty .
$$

By semi compatibility of $(P, A B)$, we get $g\left(F\left(P A B x_{2 n}, A B z ; t\right)\right) \rightarrow 0$ that is $A B z=$ $P z$.

Now taking (ii) of Lemma 3.4 with $x=z$ and $y=x_{2 n+1}$ we have

$$
\begin{aligned}
g\left(F\left(P z, Q x_{2 n+1} ; t\right)\right) \leq & \phi\left[\operatorname { m a x } \left\{g\left(F\left(Q x_{2 n+1}, S T x_{2 n+1} ; t\right)\right), g(F(P z, A B z ; t)),\right.\right. \\
& g\left(F\left(A B z, S T x_{2 n+1} ; t\right)\right), \\
& \frac{1}{2}\left(g\left(F\left(A B z, Q x_{2 n+1} ; t\right)\right)+g\left(F\left(P z, S T x_{2 n+1} ; t\right)\right)\right), \\
& \sqrt{g\left(F\left(A B z, S T x_{2 n+1} ; t\right)\right) \cdot g\left(F\left(S T x_{2 n+1}, P z ; t\right)\right)}, \\
& \left.\left.\frac{g(F(A B z, P z ; t)) \cdot g\left(F\left(P z, S T x_{2 n+1} ; t\right)\right)}{g\left(F\left(A B z, S T x_{2 n+1} ; t\right)\right)}\right\}\right]
\end{aligned}
$$

which on letting limit $n \rightarrow \infty$

$$
\begin{aligned}
g(F(P z, z ; t)) \leq & \phi[\max \{g(F(z, z ; t)), g(F(P z, A B z ; t)), g(F(A B z, z ; t)), \\
& \frac{1}{2}(g(F(A B z, z ; t))+g(F(P z, z ; t))), \\
& \sqrt{g(F(A B z, z ; t)) \cdot g(F(z, P z ; t))}, \\
& \left.\left.\frac{g(F(A B z, z ; t)) \cdot g(F(P z, z ; t))}{g(F(A B z, z ; t))}\right\}\right]
\end{aligned}
$$

i.e., $P z=z$. Thus $A B z=P z=z$. Again taking $x=B z$ and $y=x_{2 n+1}$

$$
\begin{aligned}
g\left(F\left(P B z, Q x_{2 n+1} ; t\right)\right) \leq & \phi\left[\operatorname { m a x } \left\{g\left(F\left(Q x_{2 n+1}, S T x_{2 n+1} ; t\right)\right), g(F(P B z,(A B) B z ; t)),\right.\right. \\
& g\left(F\left((A B) B z, S T x_{2 n+1} ; t\right)\right), \\
& \frac{1}{2}\left(g\left(F\left((A B) B z, Q x_{2 n+1} ; t\right)\right)+g\left(F\left(P B z, S T x_{2 n+1} ; t\right)\right)\right), \\
& \sqrt{g\left(F\left((A B) B z, S T x_{2 n+1} ; t\right)\right) \cdot g\left(F\left(S T x_{2 n+1}, P B z ; t\right)\right)} \\
& \left.\left.\frac{g(F((A B) B z, P z ; t)) \cdot g\left(F\left(P B z, S T x_{2 n+1} ; t\right)\right)}{g\left(F\left((A B) B z, S T x_{2 n+1} ; t\right)\right)}\right\}\right]
\end{aligned}
$$

on letting $n \rightarrow \infty$, we get

$$
\begin{aligned}
g(F(B z, z ; t)) \leq & \phi[\max \{g(F(z, z ; t)), g(F(B z, B z ; t)), g(F(B z, z ; t)), \\
& \frac{1}{2}(g(F(B z, z ; t))+g(F(B z, z ; t))),
\end{aligned}
$$




$$
\begin{aligned}
& \sqrt{g(F(B z, z ; t)) \cdot g(F(z, B z ; t))}, \\
&\left.\left.\frac{g(F(B z, B z ; t)) \cdot g(F(B z, z ; t))}{g(F(B z, z ; t))}\right\}\right], \\
& \text { i.e., } g(F(B z, z ; t)) \leq \phi[\max \{0,0, g(F(B z, z ; t)), 0, g(F(B z, z ; t)), 0\}] .
\end{aligned}
$$

Thus $z=A B z=P z=B z$ implies $z=A z=P z=B z$. Now since $P(X) \subseteq S T(X)$, there exists $u \in X$ such that $z=P z=S T u$. Taking (ii) of Lemma 3.4 with $x=x_{2 n}$ and $y=u$ we get

$$
\begin{aligned}
g\left(F\left(P x_{2 n}, Q u ; t\right)\right) \leq & \phi\left[\operatorname { m a x } \left\{g(F(Q u, S T u ; t)), g\left(F\left(P x_{2 n}, A B x_{2 n} ; t\right)\right),\right.\right. \\
& g\left(F\left(A B x_{2 n}, S T u ; t\right)\right), \\
& \frac{1}{2}\left(g\left(F\left(A B x_{2 n}, Q u ; t\right)\right)+g\left(F\left(P x_{2 n}, S T u ; t\right)\right)\right), \\
& \sqrt{g\left(F\left(A B x_{2 n}, S T u ; t\right)\right) \cdot g\left(F\left(S T u, P x_{2 n} ; t\right)\right)}, \\
& \left.\left.\frac{g\left(F\left(P x_{2 n}, A B x_{2 n} ; t\right)\right) \cdot g(F(Q u, S T u ; t))}{g\left(F\left(A B x_{2 n}, S T u ; t\right)\right)}\right\}\right] .
\end{aligned}
$$

Taking $n \rightarrow \infty$ we get

$$
\begin{aligned}
g(F(z, Q u ; t)) \leq & \phi[\max \{g(F(Q u, z ; t)), g(F(z, z ; t)), g(F(z, z ; t)), \\
& \frac{1}{2}(g(F(z, Q u ; t))+g(F(z, z ; t))), \\
& \sqrt{g(F(z, z ; t)) \cdot g(F(z, z ; t))}, \\
& \left.\left.\frac{g(F(z, z ; t)) \cdot g(F(Q u, z ; t))}{g(F(z, z ; t))}\right\}\right], \\
\text { i.e., } g(F(z, Q u ; t)) \leq & \phi \cdot g(F(Q u, z ; t)) .
\end{aligned}
$$

Now since the pair $(Q, S T)$ is $R$-weakly commuting, so by definition

$$
g\left(F\left(Q S x_{2 n+1}, S T Q x_{2 n+1} ; t\right)\right) \leq g\left(F\left(Q x_{2 n+1}, S T x_{2 n+1} ; t / R\right)\right)
$$

which gives $\lim _{n \rightarrow \infty} Q S T x_{2 n+1}=\lim _{n \rightarrow \infty} S T Q x_{2 n+1}=Q z$ (as $Q$ is continuous).

Now we claim that $z$ is also a fixed point of $Q$, taking $(i i)$ with $x=z$ and $y=Q x_{2 n}$

$$
\begin{aligned}
g\left(F\left(P z, Q\left(Q x_{2 n}\right) ; t\right)\right) \leq & \phi\left[\operatorname { m a x } \left\{g\left(F\left(Q\left(Q x_{2 n}\right), S T Q x_{2 n} ; t\right)\right), g(F(P z, A B z ; t)),\right.\right. \\
& g\left(F\left(A B z, S T Q x_{2 n} ; t\right)\right), \\
& \frac{1}{2}\left(g\left(F\left(A B z, Q\left(Q x_{2 n}\right) ; t\right)\right)+g\left(F\left(P z, S T Q x_{2 n} ; t\right)\right)\right), \\
& \sqrt{g\left(F\left(A B z, S T Q x_{2 n} ; t\right)\right) \cdot g\left(F\left(S T Q x_{2 n}, P z ; t\right)\right)}, \\
& \left.\left.\frac{g(F(A B z, P z ; t)) \cdot g\left(F\left(P z, S T Q x_{2 n} ; t\right)\right)}{g\left(F\left(A B z, S T Q x_{2 n} ; t\right)\right)}\right\}\right]
\end{aligned}
$$


on letting $n \rightarrow \infty$, we get

$$
\begin{aligned}
g(F(z, Q z ; t)) \leq & \phi[\max \{g(F(Q z, S T z ; t)), g(F(z, z ; t)), \\
& g(F(z, S T z ; t)), \frac{1}{2}(g(F(z, Q z ; t))+g(F(z, S T z ; t))), \\
& \sqrt{g(F(z, S T z ; t)) \cdot g(F(S T z, z ; t))}, \\
& \left.\left.\frac{g(F(z, z ; t)) \cdot g(F(z, S T z ; t))}{g(F(z, S T z ; t))}\right\}\right] \\
g(F(z, Q z ; t)) \leq & \phi[\max \{0,0, g(F(Q z, z ; t)), g(F(Q z, z ; t)), g(F(Q z, z ; t)), 0\}]
\end{aligned}
$$

i.e, $g(F(z, Q z ; t)) \leq \phi . g(F(Q z, z ; t))$ implies $Q z=z$. Thus $Q z=z=S T z$.

By taking $x=z, y=S T x_{2 n}$

$$
\begin{aligned}
g\left(F\left(P z, Q S T x_{2 n} ; t\right)\right) \leq & \phi\left[\operatorname { m a x } \left\{g\left(F\left(Q S T x_{2 n}, S T S T x_{2 n} ; t\right)\right), g(F(P z, A B z ; t)),\right.\right. \\
& g\left(F\left(A B z, S T S T x_{2 n} ; t\right)\right), \\
& \frac{1}{2}\left(g\left(F\left(A B z, Q S T x_{2 n} ; t\right)\right)+g\left(F\left(P z, S T S T x_{2 n} ; t\right)\right)\right), \\
& \sqrt{g\left(F\left(A B z, S T S T x_{2 n} ; t\right)\right) \cdot g\left(F\left(S T S T x_{2 n}, P z ; t\right)\right)}, \\
& \left.\frac{g(F(A B z, P z ; t)) \cdot g\left(F\left(P z, S T S T x_{2 n} ; t\right)\right)}{g\left(F\left(A B z, S T S T x_{2 n} ; t\right)\right)}\right\} .
\end{aligned}
$$

Taking limit $n \rightarrow \infty$ and using $(S T) T z=(T S) T z=T(S T z)=T z, Q T z=T Q z=$ $T z$ we get

$$
\begin{aligned}
g(F(z, T z ; t)) \leq & \phi[\max \{g(F(T z, z ; t)), g(F(z, z ; t)), \\
& g(F(z, T z ; t)), \frac{1}{2}(g(F(z, T z ; t))+g(F(z, T z ; t))), \\
& \sqrt{g(F(z, T z ; t)) \cdot g(F(T z, z ; t))}, \\
& \left.\left.\frac{g(F(z, z ; t)) \cdot g(F(z, T z ; t))}{g(F(z, T z ; t))}\right\}\right] \\
g(F(z, T z ; t)) \leq & \phi . g(F(T z, z ; t))
\end{aligned}
$$

i.e. $T z=z$. Thus $S T z=S z=z$, hence $z=A z=B z=S z=T z=P z=Q z$ implies $z$ is a common fixed point of $A, B, S, T, P$ and $Q$. The uniqueness of $z$ holds from inequality (ii) of Theorem 3.5.

\section{ACKNOWLEDGMENT}

The authors are greateful to the reviewer for their valuable comments and suggestions for the improvement of this paper. 


\section{REFERENCES}

1. A. Singh, R.C. Dimri \& S. Bhatt: A common fixed point theorem for weakly compatible mappings in non-Archimedean Menger PM-spaces. Mat. Vesnik 63 (2011), no. 4, 285294.

2. B. Singh \& S. Jain: Semi-compatibility, compatibility and fixed point theorems in fuzzy metric space. J. Chungcheong Math. Soc. 18 (2005), 1-22.

3. B. Schweizer \& A. Sklar: Statistical metric spaces. Pacific J. Math. 10 (1960), 313-334.

4. C. Vetro: Fixed points in weak non-Archimedean fuzzy metric spaces. Fuzzy Sets and Systems 162 (2011), 84-90.

5. G. Jungck \& B.E. Rhoades: Fixed point for set valued functions without continuity. Indian J. Pure Appl. Math. 29 (1988), 227-238.

6. G. Jungck: Compatible mappings and common fixed points. Internat. J. Math. Math. Sci. 9 (1986), 771-779.

7. G. Jungck, P.P. Murthy \& Y.J. Cho: Compatible mappings of type (A) and common fixed points. Math. Japon. 38 (1993), 381-391.

8. H.K. Pathak \& M.S. Khan: Compatible mappings of type (B) and common fixed point theorems of Gregus type. Czech. Math. J. 45 (1965), 685-698.

9. H.K. Pathak, Y.J. Cho, S.S. Chang \& S.M. Kang: Compatible mappings of type (P) and fixed point theorems in metric spaces and probabilistic metric spaces. Novi Sad J. Math. 26 (1996), 87-109.

10. H.K. Pathak, Y.J. Cho, S.M. Kang \& B. Madharia: Compatible mappings of type (C) and common fixed point theorems of Gregus type. Demonstratio Math. 31 (1998), 499-518.

11. H. Sherwood: Complete probabilistic metric spaces. Z. Wahrsch, Verw Gebiete 20 (1971), 117-128.

12. J. Achari: Fixed point theorems for a class of mappings on non-Archimedean probablistic metric spaces. Mathematica 25 (1983), 5-9.

13. M.A. Khan \& Sumitra: A common fixed point theorem in non-Archimedean Menger PM-space. Novi Sad J. Math. 39 (2009), 81-87.

14. __ A c A common fixed point theorem in 2 non-Archimedean Menger PM-space for R-weakly commuting maps of type $(p)$. Novi Sad J. Math. 38 (2008), 145-152.

15. _ _ Fixed point theorems for expansion mappings in 2 Non-Archimedean Menger PM-spaces. Novi Sad J. Math. 39 (2009), no. 2, 51-60.

16. R.A. Rashwan \& Shimaa I. Moustafa: A common fixed point theorem in nonArchimedean Menger PM-spaces. Bull. of Int. Math. Virtual Inst. 2 (2012), 141-152.

17. R.P. Pant: Common fixed points of non-commuting mappings. J. Math. Anal. Appl. 188 (1994), 436-440. 
18. R. Vasuki: Common fixed points for R-weakly commuting maps in fuzzy metric spaces. Indian J. Pure and Applied Math. 30 (1999), 419-423.

19. S.L. Singh, B.D. Pant \& Sunny Chauhan: Fixed point theorems in non-Archimedean Menger PM-spaces. J. Non-linear Anal. Opti. 3 (2012), no. 2, 153-160.

20. S.S. Chang: Fixed point theorems for single-valued and multivalued mappings in nonarchimedean Menger probabilistic metric spaces. Math. Japon. 35 (1990), 875-885.

21. S. Sessa: On a weak commutativity condition of mappings in fixed point considerations. Publications de I'Institute Mathematique (Beograd) 32 (1982), 149-153.

22. U. Mishra, H.K. Nashine, B Samet \& C. Vetro: Semi-compatible and reciprocally continuous maps in weak non-Archimedean Menger Pm-spaces. Filomat 26 (1977), no. 4, 783-792.

23. V.I. Istratescu \& Gh. Babescu: On the completion on non-Archimedean probabilistic metric spaces. Seminar de spatii metrice probabiliste, Universitatea Timisoara Nr. 17 (1979).

24. V.I. Istratescu \& G. Palea: On non-Archimedean probabilistic metric space. An. Univ. Timisoara Ser. Sti. Mat. 12 (1977), no. 2, 115-118.

25. V.I. Istratescu \& N. Crivat: On some classes of non-Archimedean probabilistic metric spaces. Seminar de spatii metrice probabiliste, Universitatea Timisoara, Nr. 121974.

26. V.I. Istratescu: Fixed point theorems for some classes of contraction mappings on nonArchimedean probablistic spaces. Publ. Math. (Debrecen) 25 (1978), 29-34.

27. ___ On some fixed point theorems with applications to the non-Archimedean Menger spaces. Attidella Acad. Naz. Lincei 58 (1975), 374-379.

28. V.M. Sehgal \& A.T. Bharucha-Reid: Fixed points of contraction mappings on probabilistic metric spaces. Math. Systems Theory 6 (1972), 97-102.

29. Y.J. Cho, H.K. Patak \& S.M. Kang: Remarks on R-weakly commuting maps and common fixed point theorems. Bull. Korean Math. Soc. 34 (1997) 247-257.

30. Y.J. Cho, K.S. Ha \& S.S. Chang: Common fixed point theorems for compatible mappings of type(A) in non-Archimedean Menger PM-spaces. Math. Japon. 46 (1997), 169-179.

${ }^{a}$ Department of Applied Sciences, nitttr (Ministry of HRD, Govt. of India) Bhopal, (M.P.) IndiA.

Email address: dk.singh1002@gmail.com

${ }^{\text {b} B a n s a l ~ I n s t i t u t e ~ o f ~ S c i e n e ~ \& ~ T e c h n o l o g y, ~ K o k t a ~ A n a n d ~ N a g a r ~ B h o p a l ~(M . P .) ~ I n d i a . ~}$ Email address: aminkhan.iqra@gmail.com 